\title{
Probiotic Potential of Fermented Foods and Their Effects on Immune System
}

\author{
İlkin Yücel Şengün 1,a,*, Damla Güney ${ }^{1, b}$

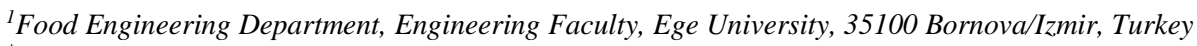

*Corresponding author

A R T I C L E IN F O A B S T R A C T

Review Article

Fermentation is known as a method that improves the nutritional value of foods in addition to preserving them. Various traditionally produced fermented foods such as yogurt, kefir, kombucha, some cheeses, sausages and pickles have probiotic potential. Hence, consumption of fermented foods plays an important role in modulating of gut microbiota and supplying positive effects on

Received : 11/09/2020

Accepted : 05/10/2021 health. With the help of beneficial microorganisms, the intestinal barrier can be improved which induces the activity of the immune cells associated to the gut. In recent years, probiotics have been used in foods in order to produce functional foods that have the potential to improve gastrointestinal health. It is known that intestinal microbiota and probiotics can interact in immune system and these

Keywords:

Fermented foods interactions provide positive health effects like reducing risk of hypertension, improving gut Probiotics Immune system Intestinal microbiota Lactic acid bacteria microbiota, regulating immune system, preventing diabetes, allergies and diarrhea. In this paper, fermented foods having probiotic potential and the effects of consuming these foods on the immune system are revised.

\section{Introduction}

Fermentation is known as a technology for the growth and metabolic activities of microorganisms which play an essential role in preservation of foods. Fermentation has been used since centuries all over the World and is considered as the most ancient, easy and inexpensive method (Sanlier et al., 2019). It has been used for different purposes such as developing new food products with various aroma profiles, achieving post-harvest preservation and improving shelf-life, preventing physiological deterioration and losses, increasing food safety and ensuring availability of foods (Patra et al., 2016; Behera and Panda, 2020). Fermentation process can be conducted either with natural indigenous microbiota or with added starter cultures, containing functional activity that alter biochemically and organoleptically the substrates to different products during process. Various foods can be fermented by lactic acid bacteria (LAB) and yeasts, while molds and Bacillus spp. may also be used for saccharification of starch or proteolysis or as secondary ripening microbiota (Marco et al., 2017). This process makes foods rich in terms of nutritional value, supplies unique flavours and textures and provides some health benefits to the consumers (Carvalho et al., 2018). Moreover, consuming fermented foods regularly has been linked to benefits on the immune system and metabolic functions. Some specific metabolites such as lactate, acetate and other short chain fatty acids found in these types of foods have anti-inflammatory and modulation properties on the gut microbiota that provide positive effects on health (Cassani et al., 2019).

In the literature, there are various studies that analyze microbiota of fermented foods. However, only limited studies have been focused on fermented foods, their probiotic potential and health benefits. This review aims to describe the probiotic potential of fermented foods and the health benefits of consuming these foods especially focusing on the effects of immune system.

\section{Microbiology of Fermented Foods}

Fermentation is a type of natural preservation of food products induced by microorganisms or enzymes that change specific substrates, commonly carbohydrates to alcohols or organic acids. Specifically, lactic acid fermentation is a basic and beneficial form of biotechnology in order to maintain and/or improve the safety degree, nutritional value, sensory qualities and shelf life of foods. Historically, various fermented products have been consumed as probiotic foods such as yogurt, kefir, kombucha, some cheeses, sausages and pickles (Montet et al., 2014). 
Table 1. Microorganisms found in some traditional fermented products

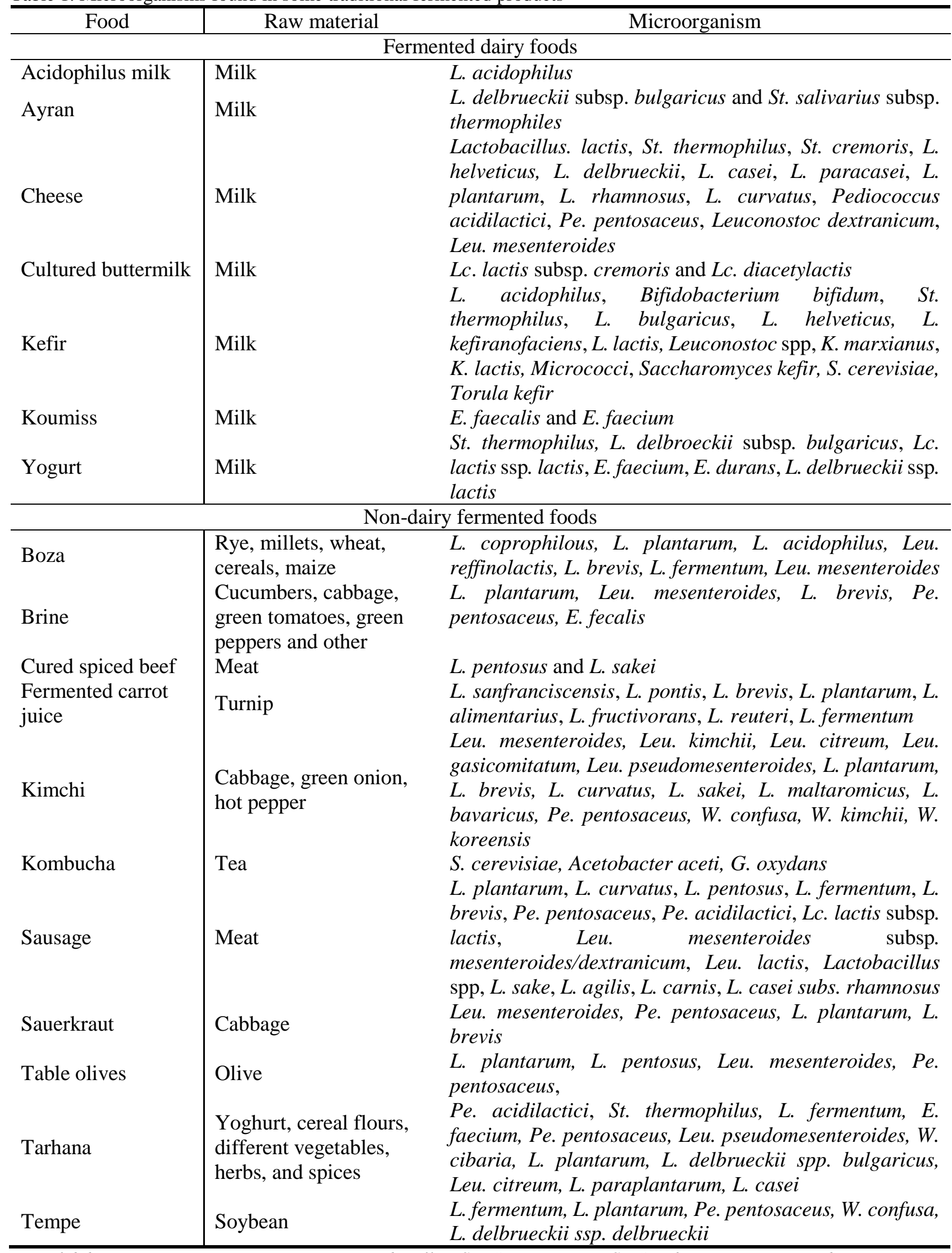

B., Bifidobacterium; E., Enterococcus; L., Lactobacillus; St., Streptococcus; S., Saccharomyces; Pe., Pediococcus; Leu., Leuconostoc; Lc., Lactococcus; K., Kluyveromyces; W., Weissella (Adapted from Evren et al., 2011; Sengün, 2011; Montet et al., 2014; Panghal et al., 2018; Adewumi, 2019) 
In table 1, some common fermented products are listed. According to their food source, fermentation was done by suitable microorganisms which means that different strains play an important role in fermentation process and supply different properties to both food and consumer. Fermentation of foods can be conducted by natural microflora of raw materials or inoculated starter cultures. LAB are commonly used as starter culture for the production of fermented foods and beverages from various raw materials. In general, LAB strains belonging to the genus Lactobacillus and Enterococcus along with bacteria of the genus Bifidobacterium show probiotic properties. Since probiotics have unique properties, they can conduct effective health benefits that make them beneficial for body functions. Hence, consuming fermented foods increase day by day because of their health benefits.

Emerging demand for the products has provided good opportunity to identify unexplored foods and beverages for isolation of LAB strains having potential role in probiotic researches (Gulbandilar et al., 2017; Patel, 2017; Adewumi, 2019). Das et al. (2020) studied with traditional fermented foods including fish, curd and rice from Meghalaya in India, in order to justify microflora of their ethnic fermented foods. A few indigenous Lactobacillus isolates (L. fermentum and L. rhamnosus spp.) from traditional fermented foods of Meghalaya were studied extensively for their technological and probiotic attributes and results showed that these indigenous isolates have high probiotic potentials. In another study, Angmo et al. (2015) identified the probiotic LAB strains from various fermented foods and beverage of Ladakh consisting of brown sourdough bread, curd, buttermilk, cottage cheese, dried cottage cheese and vegetable, and alcoholic beverages (chhang and aarak). Twenty-five LAB strains were determined in vitro for probiotic potentials based on their specific properties such as low $\mathrm{pH}$ tolerance, bile-salt resistance, lysozyme tolerance, cholesterol assimilation and exopolysaccharide production. The results showed that Lactobacillus plantarum KJ722784 had similar properties to Lactobacillus casei Shirota as reference probiotic strain. In the same study, L. plantarum KJ722784 was used as a starter culture for production of fermented milk and the results showed that viable count was greater than $7 \mathrm{log}$ $\mathrm{CFU} / \mathrm{ml}$ after 28 day storage, which makes L. plantarum KJ722784 a valuable candidate for the production of probiotic products. In a study performed by Bengoa et al. (2019), Lactobacillus paracasei strains were isolated from kefir grains in order to determine the probiotic properties and then to use it as starter cultures for the development of functional dairy products. The results of the physicochemical and immunomodulatory properties of milks fermented with L. paracasei CIDCA8339, CIDCA83123 and CIDCA83124 showed that L. paracasei CIDCA8339 can be considered as a potential probiotic strain for developing functional foods. Adesulu-Dahunsi et al. (2018) identifed the probiotic potential of 11 cerealbased foods from Nigeria and analyzed the potential of exopolysaccharide (EPS) production of LAB isolated from these fermented foods. Specifically, 3 isolates ( $L$. plantarum YO175, L. plantarum OF101 and $P$. pentosaceus OF31) showed strong probiotic properties since they had ability to withstand and adapt to gastrointestinal (GI) conditions and broad antibacterial activity. The study performed by Trzaskowska et al. (2014) showed the microbiological quality and stability, sensory acceptability and dietary value of raw-fermented sausages produced by the addition of Lactobacillus casei LOCK 0900. In the study, L. casei was inoculated in different sausage samples with $6 \log \mathrm{CFU} / \mathrm{g}$ and $7 \log \mathrm{CFU} / \mathrm{g}$. Then, microbiological quality of sausages after the fermentation and in the following 6 months storage was evaluated. The number of probiotic cells in raw-fermented sausages was nearly $9 \log \mathrm{CFU} / \mathrm{g}$ at the end of storage time. Also, the sensory evaluation results were directly correlated with the aroma of cured meat, the taste of dried meat and muscle tissue fragmentation following the fermentation. Pavli et al. (2019) studied with dry fermented sausages and considered probiotic properties of L. plantarum L125, previously isolated from a traditional Greek fermented sausage. The probiotic was added in meat batter with the commercial starter cultures ( $P$. pentosaceus and $S$. carnosus) and the microbial populations were estimated during fermentation, ripening and storage periods (at 4 and $12^{\circ} \mathrm{C}$ ). Overall, L. plantarum L125 provided equal or better characteristics to products which make it a promising probiotic starter culture in order to be used in fermented sausages. The other study analyzed probiotic potential and amylase properties of amylolytic lactic acid bacteria isolated from fermented cereal-based foods in China. Totally 132 LAB strains were isolated and among all isolates, three L. plantarum strains were confirmed to be probiotic in terms of tolerance to low $\mathrm{pH}$, bile salt resistance, and antimicrobial and aggregation activities. These three strains showed different antimicrobial activities against five food-borne pathogens and similar intrinsic and non-transmissible antibiotic susceptibilities. Researchers concluded that these three isolates are good candidates to be used as starter cultures in cereal-based probiotic foods (Xu et al., 2019). Oliveira et al. (2017) evaluated the probiotic potential of 16 yeast strains, previously isolated during the natural fermentation of green table olives, in terms of enzymatic activity, antimicrobial activity, auto-aggregation capacity, antioxidant activity and survival in gastrointestinal tract conditions. The highest antioxidant activity was observed for Saccharomyces cerevisiae, similar to the reference strain ( $S$. boulardii). Candida norvegica 7A and Galactomyces reessii 34A showed antifungal ability to Cryptococcus neoformans. Pichia guilliermondii 25A and C. norvegica 7A were the most resistant strains to the simulated digestive conditions, similar to $S$. boulardii. These results suggested that some yeast strains involved in the fermentation of table olives is potentially probiotic. Zhang et al. (2011) evaluated the probiotic properties of four different lactobacilli strains, which were L. paracasei subp. paracasei M5-L (isolated from kumiss made in local households), L. rhamnosus J10-L (isolated from fermented vegetable juice made in local households), L. casei Q8-L (isolated from yaks' yoghurt made in local households) and reference strain, L. rhamnosus GG (LGG). Also, strains were analyzed for the production of antimicrobial substances active towards some pathogens. The nature of antimicrobial substances was also investigated and shown to be dependent on the production of organic acids, particularly to lactic acid. Furthermore, the aggregation properties, adherence properties and tolerance to simulated 
gastrointestinal conditions were also investigated in in vitro tests. The results showed that M5-L, J10-L and Q8-L strains have desirable antimicrobial activity and probiotic properties as LGG. So, they have potential to be used as novel probiotic strains in the food industry. In another study, Ragul et al. (2019) studied with traditional fermented brine mango pickle and 8 selected bacterial strains were investigated in terms of antioxidative, antidiabetic and antityrosinase activities. The results of in vitro tests revealed that both intact cells and intracellular cell-free extracts of PUFSTP35 (Bacillus licheniformis KT921419), PUFSTP38 (Bacillus amyloliquefaciens KT921420) and PUFSTP39 (Bacillus subtilis KT921421) strains have significant antioxidative, antidiabetic and antityrosinase properties. According to the results, these microorganisms could be evaluated as a new starter or adjunct culture in fermentation process for better health benefits.

All these studies showed that traditional fermented foods can be considered as potential natural sources of lactic acid bacteria with probiotic characteristics. Besides, strains isolated from fermented products can be used safely for consumption and further used as a starter culture for fermentation of novel functional foods with enhanced health benefits in addition to improving the safety of food.

\section{Probiotics of Fermented Foods and Health Effects}

Some fermentative microorganisms provide functional activity which are known as probiotics. They are defined by Food and Agriculture Organization of the United Nations (FAO) and by the World Health Organization (WHO) as "live microorganisms which, when administered in adequate amounts, provide health benefits on the host (Kolady et al., 2019). Probiotics are mainly composed of LAB and Bifidobacterium. Besides, some yeasts and molds such as Saccharomyces spp. and Aspergillus spp. are also crucial probiotics (Bell et al., 2018; Boricha et al. 2019; Chugh and Kamal-Eldin, 2020). The most important factors for maintaining the adequate amount of living probiotics in the intestine are stability of intake and productivity. Also, the viability of probiotics changes their efficacy after consumption. Thus, the amount of input must be at least $10^{6}-10^{7} \mathrm{CFU} / \mathrm{g}$ in order to colonize, to survive in the gut and produce the necessary metabolites (Carvalho et al., 2018; Kim et al., 2020). Daily consumption of fermented foods has been linked to benefits on the immune system and metabolic function (Cassani et al., 2019).

Probiotic microorganisms provide various health effects such as improving or restoring the gut flora with different mechanisms involving a wide range of bioactive compounds (George Kerry et al., 2018). They create a competitive environment in gut for inhibition of the pathogens by changing the $\mathrm{pH}$ value of the environment and reducing the oxygen availability, necessary for the growth of some pathogens. Metabolites produced by probiotic strains have substantial effects on health. The main byproducts that are considered important for immune system are bacteriocin and exopolysaccharides (EPS). Bacteriocin produced by probiotics causes noncompetitive inhibition against harmful microorganisms present in the gut (Chugh and Kamal-Eldin, 2020), while
EPS play role in immune-stimulating, antioxidant and antitumor activity, cholesterol-lowering, and inhibiting or binding mutagenic agents (Sanlier et al., 2019; Novik and Savich, 2020). For example, EPS produced by Lactobacillus plantarum and Lactobacillus reuteri has an antiviral effect against human rotavirus and antiinflammatory properties (Chaisuwan et al., 2020). Moreover, probiotics synthesize nutrients like vitamins, amino acids and enzymes which are necessary for human body and enhance the bioavailability of dietary nutrients. Sufficient amounts of probiotic cells can colonize in the gastrointestinal system (GIT) and prevent many diseases such as allergic and viral diseases, Helicobacter pylori infection, diabetes, obesity, and relieve inflammation and other symptoms associated with intestinal bowel disorders. They are considerably important in stimulating host immune system (Kim and Shin, 2019; Chugh and KamalEldin, 2020; Kazemi et al., 2020). Kato-Kataoka et al. (2016) studied with milk fermented by Lactobacillus casei Shirota, and reported that consuming fermented milk helps to reduce stress related to gastrointestinal symptoms. Furthermore, gut microbial dysbiosis might be implicated in neuropsychiatric problems such as schizophrenia, bipolar disorder, and depression since gut microbiota can affect brain functions (Y1lmaz and Gökmen, 2019).

\section{Host Immune System}

In GIT of adults, the number of commensal bacteria, microbiota, in the lumen consists of approximately 400-1,000 species. They are responsible for the development and maintenance of the immune system in human body (Shimojo et al., 2005). The mucosal immune system coordinates a network of immunological responses that supply not only protection against pathogens but also tolerance to harmless antigens and bacteria. Intestinal tract is known as one of the biggest surfaces in the human body exposed to environmental antigens and to a large number of commensal bacteria. In order to live together under this antigenic pressure, the epithelium and the mucosal immune system coordinate a network of immunological response. By this way, protection against pathogens and tolerance to innocuous antigens and bacteria can be achieved (Trejo and Sanz, 2013). The gut microbiota conducts an important role in body functions. This microbiota has a unique property as an infant and consistently develops starting at childhood and then continues for a long period of time. While microbial growth increases significantly during first years of life, abnormal development of intestinal bacteria or a decrease in the complexity of the microbiota might happen. Hence, principal development patterns might be altered and the development of the immune system might be disturbed (Mousavi Khaneghah et al., 2020). More specifically, the microbiota can develop the intestinal epithelial barrier function by modulating mucin gene expression and glycosylation which affect bacterial adhesion and invasion. Some studies report that the bacteria colonizing the newborn intestine directly alter the production of salivary secretory $\operatorname{IgA}$, numbers of circulating $\operatorname{IgA}$ and $\operatorname{IgM}$ antibody producing cells and expression of innate immune receptors such as Toll- like receptors (Trejo and Sanz, 2013). Alterations in the intestinal microbiota system can influence mucosal 
immunity. Different types of diseases are correlated with unbalanced gastrointestinal microbiota such as diarrhea, allergic reactions, inflammatory diseases (atopic diseases and inflammatory bowel disease), obesity and diabetes. In order to prevent these diseases, consumption of fermented products including probiotics or consuming probiotic supplements could be better than taking antibiotics, which have many side effects. As a result, taking probiotics could improve the nonspecific intestinal barrier in gut and decrease the inflammatory condition as well as resulting in a gut immune system modulation (Watson et al., 2017; Esaiassen et al., 2018; Niu and Xiao, 2020). Probiotics, mainly LAB strains have an essential role in regulating the GIT microbiota since they can prevent the growth of opportunistic bacteria. Thus, the activity of probiotic strains in human GIT can be considered to control immune system modulation (Mousavi Khaneghah et al., 2020).

\section{Effects of Probiotics on Immune System}

Probiotics and the immune system interaction help regulation of immune responses in the human body. Health benefits of probiotics have a remarkable mechanism and it starts at the physical interaction between the probiotic and the epithelium of the human GIT. After entering the intestinal epithelium, probiotic strains can adhere the intestinal wall if they are adequate and then produce mucus used for reinforcement of the intestinal barrier. Also, with the help of this adhesion property of probiotics, pathogens can be avoided from intestinal barriers giving protective property against enteral infections, celiac disease and intestinal infection. In intestines, the epithelial cells support physical barriers which provide a natural protective system. This system gives ability to the host in order to show faster responses to activity of pathogens. Some materials produced by probiotics are linked to the protection system. One of these materials is mucins. Mucins has a glycoprotein structure and promotes protection of the mucus layer in GIT providing a physicalchemical barrier, avoiding the adherence, colonization, release of toxin and invasion of pathogens. Researchers reported that consuming foods fermented by probiotics help increasing mucins nearly $60 \%$ in the lumen (Alizadeh Behbahani et al., 2019; Mousavi Khaneghah et al., 2020). Furthermore, probiotics can increase the IgA production which can hold together the intestinal mucosa that has a crucial property for protecting the intestinal epithelium against invasions or colonization of pathogens (Zanini et al., 2007). Probiotics also enhance the activity of natural killer (NK) cells which are found in body's defense system and help increasing the mucus secretion. In order to regulate the immune system directly, probiotics release cytokines and express of co-stimulating molecules (Miller et al., 2019; Chaisuwan et al., 2020; Mohr et al., 2020).

Immune system of the body identifies intestinal microorganisms with the help of Toll-like-TLR receptors. Various biological interactions happen after adhesion of probiotics like secretion of cytokines, used for stimulation of immune system. It was reported that Lactobacillus and Bifidobacterium spp. can stimulate the immune response because of their ability to produce antibodies, activate macrophages, proliferating $\mathrm{T}$ cells and interferon (Kawashima et al., 2011; Mousavi Khaneghah et al., 2020).
Bogsan et al. (2014) studied with milk both unfermented and fermented by Bifidobacterium animalis subsp. lactis HN019 and considered the modulation of the mucosal cellular immunity. The results of the study showed that fermented milk can increase mucus and cellularity production, changes in immune pattern and preservation of mucosal epithelia. It was concluded that fermentation of milk with Bifidobacteria and/or the metabolites produced during fermentation is effective in improving host's gut. The study performed by Boricha et al. (2019) showed that Lactobacillus spp. can produce health beneficial products in GIT. Lactobacillus strains isolated from fruits, fermented foods and humans were able to survive and adhere to intestinal mucin. Also, the isolates could prevent growth of food spoilage microorganisms and various intestinal pathogens.

Bacillus coagulans which is a spore-forming probiotic bacterium is investigated by Cao et al. (2020) in terms of health effects on immune system. The research results showed that $B$. coagulans can increase intestinal digestion by producing some enzymes, which help digestion, organize microbiota, normalize both the quantitative parameters of the immune system and immune cells' functional activity and prevent the growth of pathogenic bacteria. Based on these results, B. coagulans can be considered as an important strain effective on the immune system. In another study (Jia et al., 2019), Lactobacillus salivarius AR809 isolated from human oral cavity was evaluated and characterized for its functional and immunomodulatory properties. Researchers found that $L$. salivarius could adhere epithelial cells, inhibit Staphylococcus aureus and stimulate host immunity with potentially protective effects. Also, milk could be fermented by $L$. salivarius successfully. Hence, they concluded that $L$. salivarius AR809 can be used for producing probiotic milk products and also for regulating the immune system. Lactobacillus plantarum YU was isolated from a fermented food and used in another study (Kawashima et al., 2011) for investigating its immune functions. The results showed that viable L. plantarum YU could resist to digestive juice in addition to its $\operatorname{IgA-}$ inducing activity, resulting in an enhancement of Th1 immune responses. Also, the strain could show protective activity for influenza virus infection.

\section{Conclusion}

Probiotic strains preferred in food fermentation mainly include L. casei, L. paracasei, L. plantarum, L. fermentum, L. rhamnosus, L. salivarius, Bifidobacterium animalis, $P$. pentosaceus, Bacillus licheniformis, B. coagulans, $B$. amyloliquefaciens, B. subtilis and S. boulardii, which were evaluated by various researchers as starter or adjunct cultures in fermentation process for better health benefits. Consuming probiotics have positive health effects such as reducing risk of hypertension, boosting gut microbiota, regulating immune system, and preventing diabetes, obesity, high cholesterol, diarrhea and thrombosis. In GIT, the gut microbiota performs a significant role in body functions. A relation between gut microbiota and health has been identified. There have been various impacts on health coming from microorganisms and/or its metabolites after fermentation. Hence, fermented products help 
modulating of gut microbiota and positively affect GIT. When fermented foods containing probiotics are consumed regularly, they provide positive impacts on the immune system and promote metabolic functions.

\section{Acknowledgements}

This research did not receive any specific grant from funding agencies in the public, commercial, or not-forprofit sectors.

\section{References}

Adesulu-Dahunsi AT, Jeyaram K, Sanni AI. 2018. Probiotic and technological properties of exopolysaccharide producing lactic acid bacteria isolated from cereal-based Nigerian fermented food products. Food Control, 92: 225-231. doi:10.1016/j.foodcont.2018.04.062

Adewumi GA. 2019. Health-promoting Fermented Foods. In: Melton L, Shahidi F, Varelis P. (editors), Encyclopedia of Food Chemistry. Elsevier. pp. 399-418. ISBN: 9780128140260

Alizadeh Behbahani B, Noshad M, Falah F. 2019. Inhibition of Escherichia coli adhesion to human intestinal Caco-2 cells by probiotic candidate Lactobacillus plantarum strain L15. Microbial Pathogenesis, 136: 103677. doi:10.1016/ j.micpath.2019.103677

Angmo K, Kumari A, Savitri, Bhalla TC. 2016. Probiotic characterization of lactic acid bacteria isolated from fermented foods and beverage of Ladakh. LWT - Food Science and Technology, 66: 428-435. doi:10.1016/ j.lwt.2015.10.057

Behera SS, Panda SK. 2020. Ethnic and industrial probiotic foods and beverages: efficacy and acceptance. Current Opinion in Food Science, 32: 29-36. doi:10.1016/j.cofs.2020.01.006

Bengoa AA, Iraporda C, Acurcio LB, de Cicco Sandes SH, Costa K, Guimarães GM., Arantes RME, Neumann E, Nunes AC, Nicoli JR, Garrote GL, Abraham AG. 2019. Physicochemical, immunomodulatory and safety aspects of milks fermented with Lactobacillus paracasei isolated from kefir. Food Research International, 123: 48-55. doi:10.1016/j.foodres.2019.04.041

Bell V, Ferrão J, Pimentel L, Pintado M, Fernandes T. 2018. One health, fermented foods, and gut microbiota. Foods, 7 (12): 195. doi:10.3390/foods 7120195

Bogsan CSB, Ferreira L, Maldonado C, Perdigon G, Almeida SR, Oliveira MN. 2014. Fermented or unfermented milk using Bifidobacterium animalis subsp. lactis HN019: Technological approach determines the probiotic modulation of mucosal cellular immunity. Food Research International, 64: 283-288. doi:10.1016/j.foodres.2014.05.036

Boricha AA, Shekh SL, Pithva SP, Ambalam PS, Manuel Vyas BR. 2019. In vitro evaluation of probiotic properties of Lactobacillus species of food and human origin. LWT- Food Science and Technology, 106: 201-208. doi:10.1016/ j.lwt.2019.02.021

Carvalho NM de, Costa EM, Silva S, Pimentel L, Fernandes TH, Pintado ME. 2018. Fermented foods and beverages in human diet and their influence on gut microbiota and health. Fermentation, 4(4): 90. doi:10.3390/fermentation4040090

Cao J, Yu Z, Liu W, Zhao J, Zhang H, Zhai Q, Chen W. 2020. Probiotic characteristics of Bacillus coagulans and associated implications for human health and diseases. Journal of Functional Foods, 64: 103643. doi:10.1016/j.jff.2019.103643

Cassani L, Gomez-Zavaglia A, Simal-Gandara J. 2019. Technological strategies ensuring the safe arrival of beneficial microorganisms to the gut: From food processing and storage to their passage through the gastrointestinal tract. Food Research International, 129: 108852. doi:10.1016/j.foodres.2019.108852
Chaisuwan W, Jantanasakulwong K, Wangtueai S, Phimolsiripol Y, Chaiyaso T, Techapun C, Phongthai S, You S, Regenstein JM, Seesuriyachan P. 2020. Microbial exopolysaccharides for immune enhancement: Fermentation, modifications and bioactivities. Food Bioscience, 35: 100564. doi:10.1016/j.fbio.2020.100564.

Chugh B, Kamal-Eldin A. 2020. Bioactive compounds produced by probiotics in food products. Current Opinion in Food Science, 32: 76-82. doi:10.1016/j.cofs.2020.02.003

Das S, Mishra BK, Hati S. 2020. Techno-functional characterization of indigenous Lactobacillus isolates from the traditional fermented foods of Meghalaya, India. Current Research in Food Science, 3: 9-18. doi:10.1016/j.crfs.2020.01.002

Esaiassen E, Hjerde E, Cavanagh JP, Pedersen T, Andresen JH, Rettedal SI, Stoen R, Nakstad B, Willassen NP, Klingenberg C. 2018. Effects of probiotic supplementation on the gut microbiota and antibiotic resistome development in preterm infants. Frontiers in Pediatrics, 6:347. doi:10.3389/fped.2018.00347

Evren M, Apan M, Tutkun E, Evren S. 2011. Lactic acid bacteria in traditional fermented foods. Electronic Microbiology Journal, 9(1): 11-17.

George Kerry R, Patra JK, Gouda S, Park Y, Shin HS, Das G. 2018. Benefaction of probiotics for human health: A review. Journal of Food and Drug Analysis, 26(3): 927-939. doi:10.1016/j.jfda.2018.01.002

Gülbandilar A, Okur M, Dönmez M. 2017. Properties of probiotics as a functional food. Turkish Journal of Scientific Reviews, 10(1): 44-47.

Jia GC, Che N, Xia YJ, Lai PFH, Xiong ZQ, Wang GQ, Zhang H, Ai LZ. 2019. Adhesion to pharyngeal epithelium and modulation of immune response: Lactobacillus salivarius AR809, a potential probiotic strain isolated from the human oral cavity. Journal of Dairy Science, 102(8): 6738-6749. doi:10.3168/jds.2018-16117

Kato-Kataoka A, Nishida K, Takada M, Kawai M, KikuchiHayakawa H, Suda K, Ishikawa H, Gondo Y, Shimizu K, Matsuki T, Kushiro A, Hoshi R, Watanabe O, Igarashi T, Miyazaki K, Kuwano Y, Rokutan K. 2016. Fermented milk containing Lactobacillus casei strain Shirota preserves the diversity of the gut microbiota and relieves abdominal dysfunction in healthy medical students exposed to academic stress. Applied and Environmental Microbiology, 82(12): 3649-3658. doi:10.1128/AEM.04134-15

Kazemi A, Soltani S, Ghorabi S, Keshtkar A, Daneshzad E, Nasri F, Mazloomi SM. 2020. Effect of probiotic and synbiotic supplementation on inflammatory markers in health and disease status: A systematic review and meta-analysis of clinical trials. Clinical Nutrition, 39(3): 789-819. doi:10.1016/j.clnu.2019.04.004

Kawashima T, Hayashi K, Kosaka A, Kawashima M, Igarashi T, Tsutsui H, Tsuji NM, Nishimura I, Hayashi T, Obata A. 2011. Lactobacillus plantarum strain YU from fermented foods activates Th1 and protective immune responses. International Immunopharmacology, 11(12): 2017-2024. doi:10.1016/j.intimp.2011.08.013

Kim KM, Yang SJ, Kim DS, Lee CW, Kim HY, Lee S, Choi JW, Sohn J, Shin SS, Lee S. 2020. Probiotic properties and immune-stimulating effect of the Jeju lava seawater mineralcoated probiotics. LWT- Food Science and Technology, 126: 109299. doi:10.1016/j.lwt.2020.109299

Kolady DE, Kattelmann K, Scaria J. 2019. Effects of healthrelated claims on millennials' willingness to pay for probiotics in the U.S.: Implications for regulation. Journal of Functional Foods, 60: 103434. doi:10.1016/j.jff.2019.103434 Marco ML, Heeney D, Binda S, Cifelli CJ, Cotter PD, Foligné B, Ganzle M, Kort R, Pasin G, Pihlanto A, Smid EJ, Hutkins R. 2017. Health benefits of fermented foods/ Microbiota and Beyond. Current Opinion in Biotechnology, 44: 94-102. doi/10.1016/j.copbio.2016.11.010 
Miller LE, Lehtoranta L, Lehtinen MJ. 2019. Short-term probiotic supplementation enhances cellular immune function in healthy elderly: Systematic review and meta-analysis of controlled studies. Nutrition Research, 64: 1-8. doi:10.1016/j.nutres.2018.12.011

Mohr AE, Basile AJ, Crawford MS, Sweazea KL, Carpenter KC. 2020. Probiotic supplementation has a limited effect on circulating immune and inflammatory markers in healthy adults: A systematic review of randomized controlled trials. Journal of the Academy of Nutrition and Dietetics, 120(4): 548-564. doi:10.1016/j.jand.2019.08.018

Montet D, Ray RC, Zakhia-Rozis N. 2014. Lactic Acid Fermentation of Vegetables and Fruits. In: Ray RC, Montet D (editors), Microorganisms and Fermentation of Traditional Foods. Boca Raton, USA: CRC Press. pp. 108-140. ISBN 9781482223088

Mousavi Khaneghah A, Abhari K, Es I, Soares MB, Oliveira RBA, Hosseini H, Rezaei M, Balthazar CF, Silva R, Cruz AG, Ranadheera S, Sant'Ana AS. 2020. Interactions between probiotics and pathogenic microorganisms in hosts and foods: A review. Trends in Food Science and Technology, 95: 205218. doi:10.1016/j.tifs.2019.11.022

Niu HL, Xiao JY. 2020. The efficacy and safety of probiotics in patients with irritable bowel syndrome: Evidence based on 35 randomized controlled trials. International Journal of Surgery, 75: 116-127. doi: 10.1016/j.ijsu.2020.01.142

Novik G, Savich V. 2020. Beneficial microbiota. Probiotics and pharmaceutical products in functional nutrition and medicine. Microbes and Infection, 22(1): 8-18. doi:10.1016/ j.micinf.2019.06.004

Oliveira T, Ramalhosa E, Nunes L, Pereira JA, Colla E, Pereira EL. 2017. Probiotic potential of indigenous yeasts isolated during the fermentation of table olives from Northeast of Portugal. Innovative Food Science \& Emerging Technologies, 44: 167-172. doi:10.1016/j.ifset.2017.06.003

Panghal A, Janghu S, Virkar K, Gat Y, Kumar V, Chhikara N. 2018. Food bioscience potential non-dairy probiotic products - A healthy approach. Food Bioscience, 21: 80-89. doi:10.1016/j.fbio.2017.12.003

Patel AR. 2017. Probiotic fruit and vegetable juices recent advances and future perspective. International Food Research Journal, 24(5): 1850-1857.

Patra JK, Das G, Paramithiotis S, Shin H. 2016. Kimchi and other widely consumed traditional fermented foods of Korea: a review. Front Microbiol. 7: 1-15. doi:10.3389/fmicb. 2016.01493.
Pavli FG, Argyri AA, Chorianopoulos NG, Nychas GJE, Tassou CC. 2019. Evaluation of Lactobacillus plantarum L125 strain with probiotic potential on physicochemical, microbiological and sensorial characteristics of dry-fermented sausages. LWT- Food Science and Technology, 108810. doi:10.1016/j.lwt.2019.108810

Shimojo N, Suzuki S, Campos E, Kohno Y. 2005. Effects of probiotics on the immune system and allergic diseases. Allergology International, 54(4): 515-520. doi: 10.2332/ allergolint.54.515

Sanlier N, Gökcen BB, Sezgin AC. 2019. Health benefits of fermented foods. Critical Reviews in Food Science and Nutrition, 59(3): 506-527. doi:10.1080/10408398.2017. 1383355

Sengün IY. 2011. Lactic acid bacteria used in the production of fermented foods. Biological Diversity and Conservation, 11(2): 10-14. doi:10.16194/j.cnki.31-1059/g4.2011.07.016

Trejo F, Sanz Y. 2013. Intestinal Bacteria and Probiotics: Effects on the Immune System and Impacts on Human Health. In: Calder PC, Yaqoob P. (editors), Diet, Immunity and Inflammation. Elsevier. pp. 267-268. ISBN: 9780857090379

Trząskowska M, Kołożyn-Krajewska D, Wójciak K, Dolatowski Z. 2014. Microbiological quality of raw-fermented sausages with Lactobacillus casei LOCK 0900 probiotic strain. Food Control, 35(1): 184-191. doi:10.1016/j.foodcont.2013.07.002

Y1lmaz C, Gökmen V. 2020. Neuroactive compounds in foods: Occurrence, mechanism and potential health effects. Food Research International, 128: 108744. doi:10.1016/ j.foodres.2019.108744

Watson RR, Collier RJ, Preedy VR. 2017. Nutrients in Dairy and Their Implications for Health and Disease. Academic Press. pp: 1-490. ISBN: 9780128097625

Xu Y, Zhou T, Tang H, Li X, Chen Y, Zhang L, Zhang J. 2019. Probiotic potential and amylolytic properties of lactic acid bacteria isolated from Chinese fermented cereal foods. Food Control, 107057. doi:10.1016/j.foodcont.2019.107057

Zanini K, Marzotto M, Castellazzi A, Borsari A, Dellaglio F, Torriani S. 2007. The effects of fermented milks with simple and complex probiotic mixtures on the intestinal microbiota and immune response of healthy adults and children. International Dairy Journal, 17(11): 1332-1343. doi:10.1016/j.idairyj.2007.01.017

Zhang Y, Zhang L, Du M, Yi H, Guo C, Tuo Y, Han X, Li J, Zhang L, Yang L. 2011. Antimicrobial activity against Shigella sonnei and probiotic properties of wild lactobacilli from fermented food. Microbiological Research, 167(1): 2731. doi:10.1016/j.micres.2011.02.006 